\title{
Interpretation of the Spectrum of Sn II: Experimental and Theoretical Transition Probabilities
}

\author{
A. Alonso-Medina \\ C. Colón
}

\begin{abstract}
The optical emission a from laser produced plasma generated by $1064 \mathrm{~nm}$ irradiation of $\mathrm{Sn} / \mathrm{Pb}$ alloys targets at a flux of $2 \cdot 1 \mathbf{0}^{10} \mathrm{~W} \mathrm{~cm}{ }^{2}$ was recorded and analyzed between 200 and $700 \mathrm{~nm}$. The Local Thermodynamic Equilibrium (LTE) conditions and plasma homogeneity have been checked. The analysis of Sn II was checked, with the support of parametric studies of the mixed configurations in both parities, by means of a self-consistent-field method to generate one-electron orbitals. The parametric description of the $5 \mathrm{~s} 5 \mathrm{p}^{2}$ configuration is improved by taking into account $5 \mathrm{~s}^{2} 5 \mathrm{~d}, 5 \mathrm{~s}^{2} 6 \mathrm{~d}$ and $5 \mathrm{~s}^{2} 7 \mathrm{~d}$ mixing effects. Eigenfinctions were used to derive theoretical values of the transition probabilities. Experimental transition probabilities for 36 lines of Sn II arising from the $5 \mathrm{~s}^{2} n \mathrm{~s}, 5 \mathrm{~s}^{2} n \mathrm{p}, 5 \mathrm{~s}^{2} n \mathbf{d}, 5 \mathrm{~s}^{2} n \mathrm{f}$, and $5 \mathrm{~s} 5 \mathrm{p}^{2}$ configurations of Sn II have been determined. Some values have been compared with the available data in the literature and are in a good agreement. The coincidence between several experimental and theoretical transition probabilities obtained in this work is remarked.
\end{abstract}

\section{Introduction}

The application of laser ablation for chemical element analysis of solid samples is one of the most attractive and important applications of laser produced plasmas (LPP) in science and technology. In the present work a source of LPP was used in order to obtain experimental transition probabilities of Sn II which are compared with our calculations. Both theoretical and experimental transitions are relevant not only for atomic structure research, but also for applications to astrophysics, plasma and laser physics, and also for analysis techniques. The levels of single ionized tin have been the subject of a few experimental and theoretical studies (McCormick and Sawyer [1], Wujec and Musielok [2], Wujec and Weniger [3], Miller et al. [4], Kunisz and Migdalek [5], Migdalek [6], Miller and Bengtson [7], and Marcinek and Migdalek [8]). Nevertheless elements heavier than the iron group are relevant in analysis of several peculiar stars.

In this work we present transition probabilities for 36 lines of Sn II. To our knowledge for 20 of them there are no previous values in the literature. Branching ratios for the $5 \mathrm{~s}^{2} n \mathrm{~s}$, $5 \mathrm{~s}^{2} n \mathrm{p}, 5 \mathrm{~s}^{2} n \mathrm{~d}, 5 \mathrm{~s}^{2} n \mathrm{f}$, and $5 \mathrm{~s} 5 \mathrm{p}^{2}$ lines of $\mathrm{Sn}$ II, in the range from 2000 to $7000 \AA$, have been measured by determining the emission line intensity of a laser produced plasma of alloys of lead and tin.

Absolute values of transitions probabilities were obtained from the Boltzmann plot of the line intensities of Sn II spectrum by Local Thermodynamic Equilibrium (LTE) assumption. The relative values of lines arising from the $5 \mathrm{~s}^{2} 4 \mathrm{f}^{2} \mathrm{~F}_{5 / 2}$ levels were put on an absolute scale by using the corresponding experimental lifetimes given by Gorshkov and Verolainen [9] namely 5.20.5 ns, respectively.

From the theoretical point of view we have carried out Hartree-Fock calculations including relativistic corrections and configuration interaction in a intermediate coupling scheme. The Cowan [10] code (1981) was selected for this purpose due to the ease with which it handles coupling considerations arising from the calculations of radiative transition probabilities between mixed configurations in intermediate coupling.

\section{Procedure}

\subsection{Experimental setup}

The experimental system is similar to that described in previous papers (Martinez et al. [11], Alonso-Medina [12], Colón et al. [13], Alonso-Medina et al. [14]). The experiment was carried out by emission of a plasma generated by focusing a laser beam on a sample of a $\mathrm{Sn}-\mathrm{Pb}$ alloy (with several different contents of Tin). A lens with a focal distance of $12 \mathrm{~cm}$ is used to focus, on a lead/tin target, the laser beam of a Nd:YAG laser, that generates $290 \mathrm{~mJ}$ pulses of 7 ns duration at frequency of $20 \mathrm{~Hz}$ and $10640 \AA$ wavelength. The laser irradiance on the blank was $2 \times 10^{10} \mathrm{~W} \mathrm{~cm}^{-2}$, the diameter of the standard crater was $0.5 \mathrm{~mm}$. The light emitted by the LPP was transmitted through a sapphire window to the input slit of a $1 \mathrm{~m}$ Czerny-Turner spectrometer provided with a 2400 grooves $\mathrm{mm}^{-1}$ holographic grating. The resolution of the spectroscopic system was $0.3 \AA$ in the first order. The characteristics of the apparatus are shown in Table I.

A chamber is used to generate the plasma in a vacuum or in a gas atmosphere. After a vacuum of $10^{-5}$ Torr had been attained inside the chamber, by means of a turbomolecular pump, it was filled with Argon and maintained at a pressure of 6 Torr throughout the measurement, using a small continuous flow of gas to maintain the purity of the atmosphere.

Spectra were recorded by a time-resolved optical multichannel analyzer (OMA III EG\&G) that allows the recording of spectra at a preset delay from the laser pulse and with a selected time length. Spectra were obtained at a $2.5 \mu$ s delay from the laser pulse and light was collected during $0.1 \mu \mathrm{s}$ in synchronism with the electronic trigger of the laser Q-switch. In each data acquisition period a correction was made with regard to the dark signal in the absence of the Laser Plasma. The instrumental profile of the line was determined with a precision of $97 \%$, being $0.11 \AA$ in 
second order the instrumental width (FWHM) for a wavelength of $3000 \AA$.

In the present experiment, a sample of $25 \%$ tin and $75 \%$ lead was used. For contents of this order no branching ratio dependence on concentration has been observed.

In our experimental conditions all the tabulated transitions of the Sn I and Sn II spectrum can be observed, as can those of the $\mathrm{Pb} \mathrm{I}$ and $\mathrm{Pb}$ II and some of the Ar I and Ar II. Various emission spectra were recorded in order to obtain the transition probabilities with regard to a statistical uncertainty of $3 \%$.

Figure 1 displays a typical section of measured spectrum in the 5535 to $5620 \AA$ wavelength range obtained in the above mentioned experimental conditions.

To calibrate the spectral response of the system in the wavelength range studied $(2000-7000 \AA)$, a deuterium lamp was used for the $2000-4000 \AA$ range, and a tungsten lamp was used for the range $3500-7000 \AA$. The instrumental profile was predetermined by observation of various narrow lines emitted by hollow cathode lamps.

The same experimental system was used to study the homogeneity of the plasma in order to have spatial resolution, and determine where the different atomic species of tin are located in the plasma. The light was focused by means of a lens on a $1 \mathrm{~mm}$ light guide being able to select the point of the plasma from which the light emission was observed. The measurements were taken by scanning

\section{Table I. Experimental apparatus and characteristics.}

\begin{tabular}{ll}
\hline Q-switched Nd:YAG Laser & Quantel YG 585 \\
Pulsewidth & $7 \mathrm{~ns}$ \\
Pulse energy & $290 \mathrm{~mJ}$ \\
Wavelength & $10640 \mathrm{~A}$ \\
Repetition rate & $20 \mathrm{~Hz}$ \\
& \\
Spectrometer & Spex 1704 \\
Type & $1 \mathrm{~m}$, Czerny-Turner \\
Grating & 2400 grooves/mm \\
Slit & $50 \mu \mathrm{m}$ \\
Detection system & EG\&G OMA III (model 1461) \\
Number of channels & 1024 \\
Minimum gate width & $100 \mathrm{~ns}$ \\
& \\
Fiber-optic cable & $\mathrm{UV}$ fused silica, incoherent bundle \\
Length & $5 \mathrm{~m}$ \\
Diameter & $1 \mathrm{~mm}$ \\
\hline
\end{tabular}

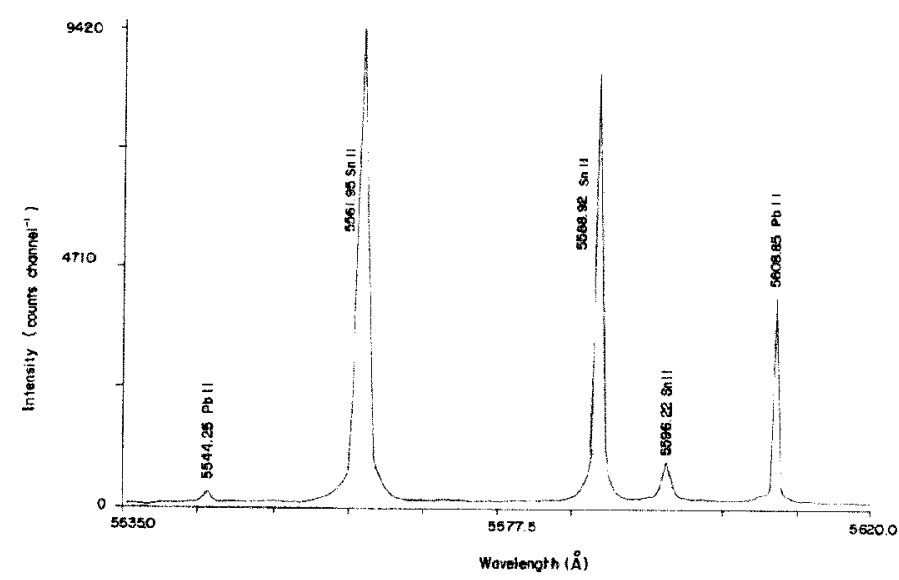

Fig. I. Section of typical Sn II spectra at 6 Torr Ar ( $2.5 \mu$ s delay time). the plasma emission in two perpendicular directions: through the axis of the plasma, with a distance from the target in the 0.25 to $2.75 \mathrm{~mm}$ range, to study the evolution of the plasma in space; and parallel to the surface of the target with a radial distance in the range of 0.0 to $1.12 \mathrm{~mm}$.

The different spectra in these scannings were stored in a computer for further analysis, which was made by fitting the observed line shapes to numerically generated Voigt profiles. Local profiles were obtained after Abel inversion of the integrated intensity, [15]. The fitting of the observed profiles provide the total intensity very accurately, as well as the broadening of the spectral lines.

\subsubsection{Determination of the plasma temperature and the electron densities}

As is well known for an optically thin plasma, the relative intensities $I_{i j}$ of the lines emitted from a given state of excitation can be used to calculate the electron temperature, if the $A_{i j}$ transition probabilities are known, by the expression:

$I_{i j}=\frac{A_{i j} g_{i}}{U(T)} N \exp \left(\frac{-E_{i}}{k T}\right)$,

for a transition from a higher state $i$ to a lower state $j . I_{i j}$ is the relative intensity, $E_{i}$ and $g_{i}$ are the energy and statistical weight of level $i, U(T)$ is the atomic species partition function, $N$ the total density of emitting atoms, $k$ the Boltzmann constant and $T$ the temperature. If we were to plot $\ln \left(I_{i j} / g_{i} A_{i j}\right)$ vs. $E_{i}$, a Boltzmann plot, the resulting straight line would have a slope $-1 / k T$, and therefore the temperature can be obtained without having to know the total density of atoms or the atomic species partition function.

The well-known Sn II transitions used for the determination of the excitation temperature by Boltzmann plot are shown in Table II. The energies of the different levels are those of Moore [16] and the transition probabilities $A_{i j}$ required are taken from McCormick and Sawyer [1] and Miller et al. [4]. Figure 2 displays a Boltzmann plot from which a value of $(11033 \pm 450) \mathrm{K}$ for $=6.68 \mathrm{eV}$ was obtained from the electron temperature.

As a confirmation we also obtained the plasma temperature from lines of $\mathrm{PbII}$ (using the $5608.9 \AA, 6660.0 \AA$,

Table II. Additional spectroscopic data of Sn II and $\mathrm{Pb} I I$ employed for temperature determination.

\begin{tabular}{|c|c|c|c|c|}
\hline $\begin{array}{l}\text { Atomic } \\
\text { species }\end{array}$ & $\begin{array}{l}\text { Wavelength } \\
(\AA)\end{array}$ & $\begin{array}{l}\text { Energy (eV) } \\
\text { (upper level) }\end{array}$ & $\begin{array}{l}g_{i} \\
\text { (upper level) }\end{array}$ & $\begin{array}{l}A_{i j} \\
\left(10^{7} \mathrm{~s}^{1}\right)\end{array}$ \\
\hline \multirow[t]{5}{*}{ Sn II } & 2368.3 & 5.76 & 2 & $0.044 \pm 40 \%$ \\
\hline & 6844.2 & 8.86 & 2 & $6.1 \pm 30 \%$ \\
\hline & 6453.6 & 8.97 & 4 & $6.6 \pm 33 \%{ }^{b}$ \\
\hline & 5562.0 & 11.20 & 6 & $13.0 \pm 25 \%$ \\
\hline & 3575.4 & 12.44 & 6 & $1.3 \pm 40 \%$ \\
\hline \multirow[t]{5}{*}{$\mathrm{Pb}$ II } & 6660.0 & 9.33 & 2 & $5.7 \pm 0.5^{\mathrm{c}}$ \\
\hline & 5608.9 & 9.58 & 4 & $8.4 \pm 0.8^{\mathrm{c}}$ \\
\hline & 4386.5 & 11.47 & 6 & $15.6 \pm 1.6^{\mathrm{c}}$ \\
\hline & 3718.3 & 12.57 & 2 & $1.2 \pm 0.2^{\mathrm{c}}$ \\
\hline & 3713.9 & 12.92 & 6 & $4.2 \pm 0.6^{\mathrm{c}}$ \\
\hline
\end{tabular}




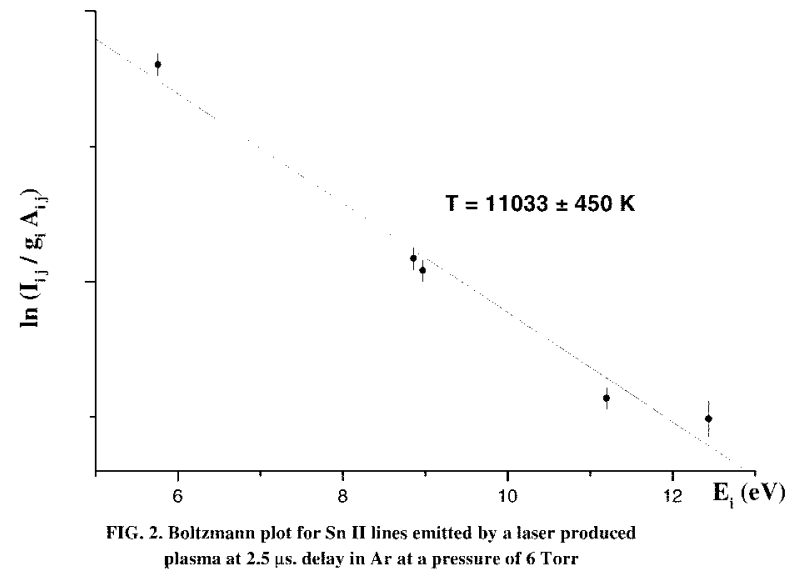

Fig. 2. Boltzmann plot for Sn II lines emitted by a laser produced plasma at $2.5 \mu$ s delay in $\mathrm{Ar}$ at a pressure of 6 Torr.

$4386.5 \AA, 3718.3 \AA$, and $3713.9 \AA$ transitions; transition probabilities were taken from Alonso-Medina [12]) with a value of $(10900 \pm 300) \mathrm{K}$ for $\Delta E=3.34 \mathrm{eV}$. This value is totally compatible with the result obtained from the $\mathrm{Sn}$ II lines.

The electron density, $N_{\mathrm{e}}$, of the plasma investigated have been obtained by comparing the Stark broadening for several transitions with those of other authors, using the McWhirter [17] and Sobelmann [18] expression,

$\Delta=2 \omega\left(\frac{N_{\mathrm{e}}}{10^{16}}\right)\left[1+1 / 75 A\left(\frac{N_{\mathrm{e}}}{10^{16}}\right)^{1 / 4}\left(1-1.2 N_{\mathrm{D}}^{-1 / 3}\right)\right]$

were $\Delta \lambda$ is the Full Width at Half Maximum (FWHM) of the transition considered, $\omega$ is the electron impact parameter, $A$ is the ion-broadening parameter. $N_{\mathrm{D}}$ is the number of particles in the Debye sphere and was estimated by $N_{\mathrm{D}}=1,38 \cdot 10^{3} T^{3 / 2} N_{\mathrm{e}}^{-1 / 2}$ which is well above the limit $N_{\mathrm{D}}=2$ of the Debye approximation for correlation effects, $[18,19]$. The value obtained was $\cong 6 \cdot 10^{16} \mathrm{~cm}^{-3}$.

The values of the electron densities from different spectral lines are in good agreement. The electron impact parameter for these lines was obtained by extrapolation in Table III which displays the values obtained with the $5332.4 \AA$, $5588.8 \AA, 3575.4 \AA$ and $3283.1 \AA$ lines of Sn II, with 4244.9 $\AA$, $5544.3 \AA$, and $5608.9 \AA$ lines of $\mathrm{Pb}$ II. The electron densities obtained from the Stark broadening may be considered reliable because the other broadening mechanisms considered in this study barely account for $3 \%$ of the total broadening value.

These values are sufficient to assume LTE for the population of the studied levels according to the criterion of McWhirter [17]:

$N_{\mathrm{e}}\left(\mathrm{cm}^{-3}\right) \geq 1.6 \cdot 10^{12} \sqrt{T}(\Delta E)^{3}$,

where $\Delta E$, in $\mathrm{eV}$, is the energy difference between both configurations and $T$, in $\mathrm{K}$, the plasma temperature, and $N_{\mathrm{e}}$ the lower limit of the electron density necessary to maintain the populations of the energy level at $10 \%$ of the LTE by collision, in competition with the radiative processes.

In this work self-absorption effects, estimated by absorption coefficient calculations [21], turned out to be lower than $3 \%$ for the most intense lines, and therefore the plasma can be considered optically thin.

In order to determine the change of the temperature and the electron density in different regions of the plasma several $\mathrm{Sn} \mathrm{II}$ and $\mathrm{Pb}$ II lines had been used. These results indicate a satisfactory homogeneity for temperature and electron density. Deviations from the average are less than $10 \%$ for $N_{\mathrm{e}}$ and less than $5 \%$ for $T$ in a region measuring approximately $2.5 \mathrm{~mm}$ in size corresponding to $95 \%$ of the emission of light; similar results have been obtained in other LPP experiments.

\subsection{Theoretical calculations}

Singly ionized tin involves a single optical electron whose energy levels are well separated. This structure must allow us to obtain a suitable comparison between experimental and theoretical results with relativistic central field calculations using the $L S$ coupling scheme. Nevertheless this scheme is not enough to describe the $5 \mathrm{~s} 5 \mathrm{p}^{2}$ configuration [8] and a more detailed description is necessary to take into account some results. For comparison with and interpretation of experimental results we have obtained theoretical

Table III. Electron density of plasma (6 Torr of Ar, delay time of $2.5 \mu \mathrm{s}$ ).

\begin{tabular}{|c|c|c|c|c|}
\hline Sn II Transition & $\begin{array}{l}\text { Wavelength } \\
(\AA)\end{array}$ & $\begin{array}{l}\text { Temperature } \\
\left(\times 10^{3} \mathrm{~K}\right)\end{array}$ & $\begin{array}{l}\mathrm{N}_{\mathrm{e}}=10^{17} \mathrm{~cm}^{3} \\
\omega_{\exp }(\AA) \pm 15 \%\end{array}$ & $\begin{array}{l}\text { This Work } \\
\mathrm{N}_{\mathrm{e}}\left(\times 10^{16} \mathrm{~cm}^{3}\right) \\
11033 \mathrm{~K}\end{array}$ \\
\hline $6 \mathrm{~d}^{2} \mathbf{D}_{3 / 2} \rightarrow 6 \mathrm{p}^{2} \mathbf{P}_{1 / 2}$ & 5332.4 & 11.6 & $5.3^{\mathrm{a}}$ & $6.4 \pm 1.0$ \\
\hline $4 \mathrm{f}^{2} \mathbf{F}_{5 / 2} 5 \mathrm{~d} \rightarrow{ }^{2} \mathbf{D}_{3 / 2}$ & 5588.9 & 11.6 & $3.8^{\mathrm{a}}$ & $5.0 \pm 1.2$ \\
\hline $4 \mathrm{f}^{2} \mathbf{F}_{5 / 2} 5 \mathrm{p}^{2} \rightarrow{ }^{2} \mathbf{D}_{3 / 2}$ & 3283.2 & 11.6 & $2.3^{\mathrm{a}}$ & $5.2 \pm 1.0$ \\
\hline $7 \mathrm{~d}^{2} \mathbf{D}_{3 / 2} \rightarrow 6 \mathrm{p}^{2} \mathbf{P}_{3 / 2}$ & 3575.5 & 11.6 & $3.0^{\mathrm{a}}$ & $7.2 \pm 1.3$ \\
\hline $\mathrm{Pb}$ II Transition & $\begin{array}{l}\text { Wavelength } \\
(\AA)\end{array}$ & $\begin{array}{l}\text { Temperature } \\
\left(\times 10^{3} \mathrm{~K}\right)\end{array}$ & $\begin{array}{l}\mathrm{N}_{\mathrm{e}}=10^{17} \mathrm{~cm}^{3} \\
\omega_{\exp }(\AA) \pm 15 \%\end{array}$ & $\begin{array}{l}\text { This Work } \\
\mathrm{N}_{\mathrm{e}}\left(\times 10^{16} \mathrm{~cm}^{3}\right) \\
11033 \mathrm{~K}\end{array}$ \\
\hline $5 \mathrm{f}^{2} \mathrm{~F}_{7 / 2} \rightarrow 6 \mathrm{~d}^{2} \mathrm{D}_{5 / 2}$ & 4244.9 & 11 & $1.69^{\mathrm{b}}$ & $6.8 \pm 1.0$ \\
\hline $7 \mathrm{~d}^{2} \mathbf{D}_{5 / 2} \rightarrow 7 \mathrm{p}^{2} \mathbf{P}_{3 / 5}$ & 5544.3 & 11 & $3.60^{\mathrm{b}}$ & $6.4 \pm 0.9$ \\
\hline $7 \mathrm{p}^{2} \mathbf{P}_{3 / 2} \rightarrow 7 \mathrm{~s}^{2} \mathrm{~S}_{1 / 2}$ & 5608.9 & 11 & $1.96^{\mathrm{b}}$ & $5.8 \pm 0.9$ \\
\hline
\end{tabular}

${ }^{a}$ Miller et al. [4].

${ }^{\mathrm{b}}$ Puric et al. [20]. 
values of transition probabilities in intermediate coupling (IC) and using ab initio relativistic Hartree-Fock (HFR) calculations.

For the IC calculations we used the standard method of least-square fitting of experimental energy levels by means of computer codes from Cowan [10]. In this way we obtained the LS composition of each level and the degree of configuration mixing, when we consider their interaction. For the HFR calculations the Cowan [10] computer code provide us with the radial parts for determination of transition probabilities and initial estimated of the parameters for the IC fittings. The radial integrals for calculations of transition probabilities were obtained from the HFR wavefunctions.

\section{Results and discussion}

\section{1. $5 s 5 p^{2}-5 s^{2} 5 d$ configuration interaction}

Levels from Sn II $5 s^{2} 5 d$ and $5 s 5 p^{2}$ configurations present some anomalies whose interpretation will be discussed here. The energy level positions expected for these configurations in absence of interactions can be observed on the left hand

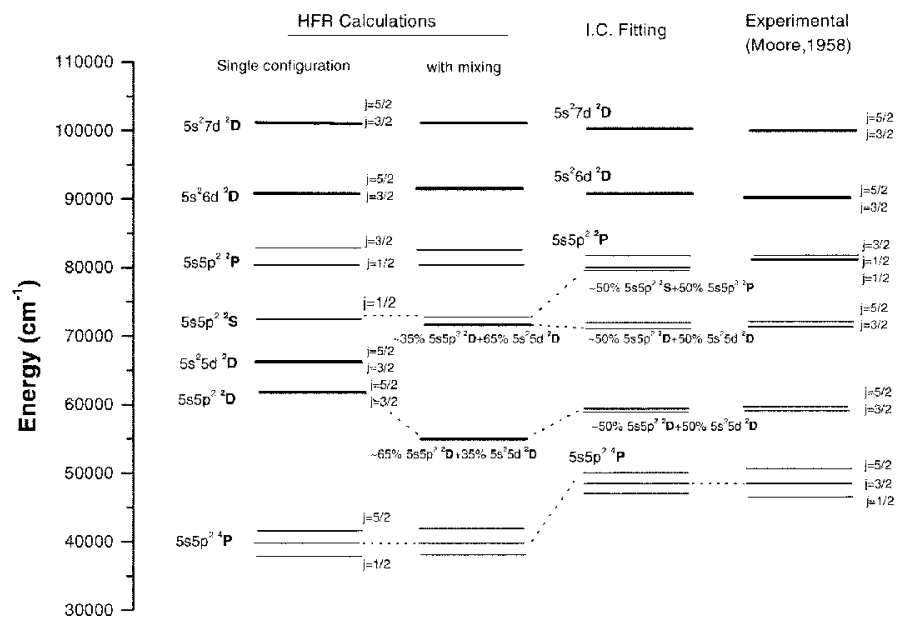

Fig. 3. Effects of $5 s^{2} 5 d-5 s 5 p^{2}$ configuration interaction. section of Fig. 3. The values of this first diagram come from a theoretical HFR calculation of separated $5 \mathrm{~s} 5 \mathrm{p}^{2}-5 \mathrm{~s}^{2} 5 \mathrm{~d}-$ $5 s^{2} 6 d-5 s^{2} 7 d$ configurations with no consideration of their interaction. A comparison between these results and the experimental values ( the right hand section of Fig. 3) shows a large discrepancy.

The second diagram in Fig. 3 shows the effect of allowing for configuration interaction in the HFR calculation. The basis set used in present calculations consists in 7 configurations of even parity namely $5 s 5 p^{2}-5 s^{2} 5 d-5 s^{2} 6 d-5 s^{2} 7 d-$ $5 s^{2} 6 s-5 s^{2} 7 s-5 s^{2} 8 s$. The effect of "energy level repulsion" is clearly significant between $5 \mathrm{~s} 5 \mathrm{p}^{2}{ }^{2} \mathrm{D}$ levels and $5 \mathrm{~s}^{2} 5 \mathrm{~d}^{2} \mathrm{D}$, and as is well know [8] very heavy mixing occurs for them.

Finally, the third diagram of this figure presents the fitting of experimental energy levels in the IC calculations. It must be noticed that:

- Although the relative position of the $5 s^{2} 5 d{ }^{4} \mathbf{P}$ multiplet energy are close to the experimental, the fine level splitting presents a marked discrepancy around $900 \mathrm{~cm}^{-1}$.

- The theoretical energy levels of ${ }^{2} \mathbf{D}$ corresponding to the $5 s 5 p^{2}-5 s^{2} 5 d$ configurations are close to the experimental. Its heavy mixing has increased and according to this interpretation the lower ${ }^{2} \mathbf{D}_{\mathbf{3} / \mathbf{2}}$ should be more properly assigned to the $5 \mathrm{~s}^{2} 5 \mathrm{~d}$ configuration and not to the $5 \mathrm{~s} 5 \mathrm{p}^{2}$ as is usually done.

- The $5 \mathrm{~s} 5 \mathrm{p}^{2}{ }^{\mathbf{2}} \mathbf{S}_{\mathbf{1 / 2}}$ and $5 \mathrm{~s} 5 \mathrm{p}^{2}{ }^{\mathbf{2}} \mathbf{P}_{\mathbf{1 / 2}}$ levels present a marked mixing more relevant than in the pure HFR calculations.

The above mentioned discrepancies between experimental and theoretical energy levels suggested the presence of other configurations in interaction. In order to eliminate them we have consider the 20 even configurations suggested by Marcinek and Migdalek [8]. Considerations of some of these configurations and a HFR calculation taking into account the complete set was unfruitful. Attempts to carry out a least-square fitting including these configurations resulted in no reasonable improvement while presenting difficulties due to the increase in the number of parameters above the number of experimentally observed energy levels.

Additional details for the above calculations are given in Tables IV and VI. The former includes the values found for the different parameters involved in the IC calculations, whereas the latter displays the transition probabilities. These will be described in the next section.

Table IV. Parameters resulting from the least-squares fitting of the Intermediate Coupling calculations (values in parenthesis are ab initio HFR results).

\begin{tabular}{ll}
\hline Configuration & \\
\hline $5 \mathrm{~s}^{2} 6 \mathrm{~s}$ & $E_{\mathrm{av}}=54690(55530)$ \\
$5 \mathrm{~s}^{2} 7 \mathrm{~s}$ & $E_{\mathrm{av}}=83518(84706)$ \\
$5 \mathrm{~s}^{2} 8 \mathrm{~s}$ & $E_{\mathrm{av}}=95831(96697)$ \\
$5 \mathrm{~s} 5 \mathrm{p}^{2}$ & $E_{\mathrm{av}}=60816(55557)$
\end{tabular}

\begin{tabular}{ll}
$5 \mathrm{~s}^{2} 5 \mathrm{~d}$ & $E_{\mathrm{av}}=63064(64159)$ \\
$5 \mathrm{~s}^{2} 6 \mathrm{~d}$ & $E_{\mathrm{av}}=87069(88239)$ \\
$5 \mathrm{~s}^{2} 7 \mathrm{~d}$ & $E_{\mathrm{av}}=97315(98460)$ \\
\hline
\end{tabular}

$$
\begin{aligned}
& F^{2}(5 \mathrm{p}, 5 \mathrm{p})=33099(31303) \\
& G^{1}(5 \mathrm{~s}, 5 \mathrm{p})=32425(41338) \\
& \alpha=-271 \\
& R^{1}(5 \mathrm{~s}, 5 \mathrm{p}, 5 \mathrm{~s}, 5 \mathrm{~d})=17621(23376) \\
& R^{1}(5 \mathrm{~s}, 5 \mathrm{p}, 5 \mathrm{~s}, 6 \mathrm{~d})=11178(12142) \\
& R^{1}(5 \mathrm{~s}, 5 \mathrm{p}, 5 \mathrm{~s}, 7 \mathrm{~d})=10448(8065) \\
& R^{1}(5 \mathrm{~s}, 5 \mathrm{p}, 5 \mathrm{~s}, 6 \mathrm{~s})=-4845(-2352) \\
& R^{1}(5 \mathrm{~s}, 5 \mathrm{p}, 5 \mathrm{~s}, 7 \mathrm{~s})=-1920(-1379) \\
& R^{1}(5 \mathrm{~s}, 5 \mathrm{p}, 5 \mathrm{~s}, 8 \mathrm{~s})=1444(-960)
\end{aligned}
$$$$
\zeta_{5 \mathrm{P}}=1436(2682)
$$

$\zeta_{5 \mathrm{~d}}=456(67)$
$\zeta_{6 \mathrm{~d}}=46(27)$
$\zeta_{7 \mathrm{~d}}=22(14)$ 
Table V. Comparisons of experimentally determined transitions probabilities of Sn II with others results.

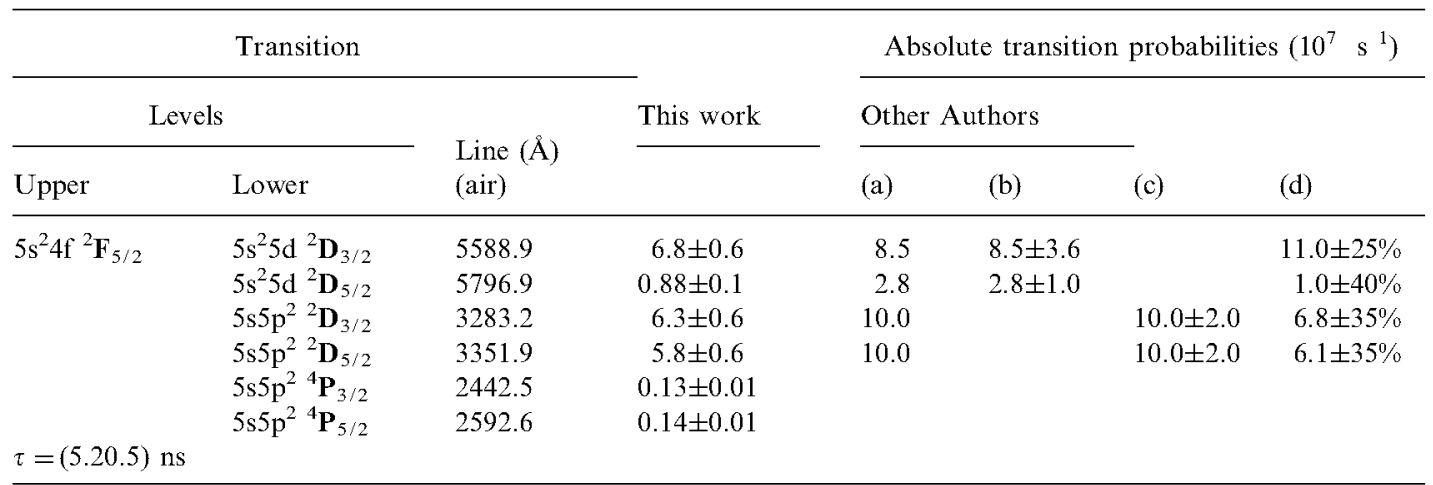

${ }^{\mathrm{a}}$ McCormick and Sawyer [1].

${ }^{\mathrm{b}}$ Wujec and Weniger [3].

${ }^{\mathrm{e}}$ Wujec and Musielok [2].

${ }^{\mathrm{d}}$ Miller et al. [4].

Table VI. Experimental and theoretical transition probabilities of some lines of Sn II.

\begin{tabular}{|c|c|c|c|c|c|c|c|c|c|}
\hline & & & \multicolumn{7}{|c|}{ Absolute transition probabilities $\left(10^{7} \mathrm{~s}^{1}\right)$} \\
\hline \multicolumn{3}{|c|}{ Transition } & \multicolumn{3}{|c|}{ This work } & & & & \\
\hline \multicolumn{2}{|c|}{ Levels } & \multirow{2}{*}{$\begin{array}{l}\text { Line }(\AA) \\
\text { (air) }\end{array}$} & \multirow[b]{2}{*}{ Experimental } & \multicolumn{2}{|c|}{ Theory } & \multicolumn{4}{|c|}{ Other Authors } \\
\hline Upper & Lower & & & HFR & FIT & (a) & (b) & (c) & (d) \\
\hline $5 \mathrm{~s}^{2} 6 \mathrm{p}^{2} \mathbf{P}_{1 / 2}$ & $5 \mathrm{~s}^{2} 6 \mathrm{~s}^{2} \mathbf{S}_{1 / 2}$ & 6844.1 & $5.6 \pm 1.1$ & 6.8 & 6.8 & 6.6 & 6.62 .1 & & $6.130 \%$ \\
\hline \multirow{2}{*}{$5 \mathrm{~s}^{2} 6 \mathrm{p}^{2} \mathbf{P}_{3 / 2}$} & $5 \mathrm{~s}^{2} 6 \mathrm{~s}^{2} \mathbf{S}_{1 / 2}$ & 6453.5 & $5.2 \pm 1.0$ & 8.0 & 7.5 & 12.0 & 12.13 .6 & & $6.635 \%$ \\
\hline & $5 \mathrm{~s} 5 \mathrm{p}^{2}{ }^{4} \mathbf{P}_{5 / 2}$ & 4618.3 & $0.059 \pm 0.010$ & 0.045 & 0.0096 & & & & $0.05430 \%$ \\
\hline \multirow{2}{*}{$5 s^{2} 7 p^{2} \mathbf{P}_{1 / 2}$} & $5 s 5 p^{22} \mathbf{D}_{3 / 2}$ & 3023.9 & $0.29 \pm 005$ & 3.1 & 1.1 & & & & \\
\hline & $5 \mathrm{~s}^{2} 5 \mathrm{~d}^{2} \mathbf{D}_{3 / 2}$ & 4877.2 & $0.13 \pm 0.05$ & 0.55 & 0.45 & & & & \\
\hline \multirow[t]{3}{*}{$5 s^{2} 7 p^{2} \mathbf{P}_{3 / 2}$} & $5 \mathrm{~s} 5 \mathrm{p}^{22} \mathbf{D}_{3 / 2}$ & 2991.0 & $0.074 \pm 0.01$ & 0.31 & 0.12 & & & & \\
\hline & $5 s 5 p^{22} \mathbf{D}_{5 / 2}$ & 3047.5 & $0.29 \pm 0.04$ & 2.8 & 1.1 & & & & \\
\hline & $5 \mathrm{~s}^{2} 5 \mathrm{~d}^{2} \mathbf{D}_{5 / 2}$ & 4944.3 & $0.11 \pm 0.03$ & 0.52 & 0.38 & & & & \\
\hline \multirow[t]{2}{*}{$5 \mathrm{~s}^{2} 6 \mathrm{~d}^{2} \mathrm{D}_{3 / 2}$} & $5 s^{2} 6 p^{2} \mathbf{P}_{1 / 2}$ & 5332.4 & $5.8 \pm 1.0$ & 10.7 & 10.5 & 8.6 & 8.63 .1 & & $12.030 \%$ \\
\hline & $5 \mathrm{~s}^{2} 6 \mathrm{p}^{2} \mathbf{P}_{3 / 2}$ & 5596.3 & $1.3 \pm 0.2$ & 1.9 & 1.8 & 1.5 & 1.50 .5 & & $1.340 \%$ \\
\hline $5 \mathrm{~s}^{2} 6 \mathrm{~d}^{2} \mathbf{D}_{5 / 2}$ & $5 \mathrm{~s}^{2} 6 \mathrm{p}^{2} \mathbf{P}_{3 / 2}$ & 5561.9 & $9.1 \pm 1.9$ & 11.5 & 11.8 & 12.0 & 11.84 .0 & & $13.025 \%$ \\
\hline \multirow{2}{*}{$5 \mathrm{~s}^{2} 7 \mathrm{~d}^{2} \mathbf{D}_{3 / 2}$} & $5 \mathrm{~s}^{2} 6 \mathrm{p}^{2} \mathbf{P}_{1 / 2}$ & 3472.5 & $1.4 \pm 0.2$ & 4.6 & 4.9 & 1.6 & & $1.6 \pm 3.0$ & $1.040 \%$ \\
\hline & $5 \mathrm{~s}^{2} 6 \mathrm{p}^{2} \mathbf{P}_{3 / 2}$ & 3582.4 & $0.35 \pm 0.08$ & 0.86 & 0.92 & & & & \\
\hline $5 \mathrm{~s}^{2} 7 \mathrm{~d}^{2} \mathbf{D}_{5 / 2}$ & $5 \mathrm{~s}^{2} 6 \mathrm{p}^{2} \mathbf{P}_{3 / 2}$ & 3575.5 & $2.7 \pm 0.5$ & 5.2 & 5.6 & 1.3 & & $1.3 \pm 3.0$ & $1.340 \%$ \\
\hline $5 \mathrm{~s}^{2} 7 \mathrm{~s}^{2} \mathbf{S}_{1 / 2}$ & $5 s^{2} 6 p^{2} \mathbf{P}_{1 / 2}$ & 6761.4 & $4.2 \pm 1.0$ & 4.1 & 3.8 & 3.2 & 3.21 .0 & & $3.735 \%$ \\
\hline \multirow{2}{*}{$5 s^{2} 8 s^{2} \mathbf{S}_{1 / 2}$} & $5 s^{2} 6 p^{2} \mathbf{P}_{1 / 2}$ & 3715.2 & $1.8 \pm 0.3$ & 1.6 & 1.6 & & & & \\
\hline & $5 s^{2} 6 p^{2} \mathbf{P}_{3 / 2}$ & 3841.4 & $2.5 \pm 0.5$ & 2.9 & 2.9 & & & & \\
\hline \multirow{6}{*}{$5 \mathrm{~s}^{2} 4 \mathrm{f}^{2} \mathbf{F}_{5 / 2}$} & $5 \mathrm{~s}^{2} 5 \mathrm{~d}^{2} \mathbf{D}_{3 / 2}$ & 5588.9 & $6.7 \pm 1.2$ & 10.8 & 9.6 & 8.5 & 8.53 .6 & & $11.025 \%$ \\
\hline & $5 \mathrm{~s}^{2} 5 \mathrm{~d}^{2} \mathbf{D}_{5 / 2}$ & 5796.9 & $0.87 \pm 0.2$ & 0.75 & 0.65 & 2.8 & 2.81 .0 & & $1.040 \%$ \\
\hline & $5 s 5 p^{22} D_{3 / 2}$ & 3283.2 & $6.3 \pm 1.2$ & 17.4 & 20.5 & 10.0 & & $10.0 \pm 2.0$ & $6.835 \%$ \\
\hline & $5 s 5 \mathrm{p}^{2}{ }^{2} \mathbf{D}_{5 / 2}$ & 3351.9 & $5.7 \pm 1.0$ & 1.2 & 1.2 & 10.0 & & $10.0 \pm 2.0$ & $6.135 \%$ \\
\hline & $5 \mathrm{~s} 5 \mathrm{p}^{2}{ }^{4} \mathbf{P}_{3 / 2}$ & 2442.5 & $0.13 \pm 0.03$ & & & & & & \\
\hline & $5 s 5 p^{2}{ }^{4} \mathbf{P}_{5 / 2}$ & 2592.6 & $0.14 \pm 0.03$ & & & & & & \\
\hline \multirow[t]{3}{*}{$5 \mathrm{~s}^{2} 4 \mathrm{f}^{2} \mathbf{F}_{7 / 2}$} & $5 \mathrm{~s}^{2} 5 \mathrm{~d}^{2} \mathbf{D}_{5 / 2}$ & 5798.9 & $8.1 \pm 1.6$ & 11.2 & 9.7 & 8.1 & 8.12 .9 & & $7.435 \%$ \\
\hline & $5 s 5 p^{22} \mathbf{D}_{5 / 2}$ & 3351.3 & $6.0 \pm 1.3$ & 18.3 & 18.1 & & & & \\
\hline & ${ }_{5 s} 5 p^{2}{ }^{4} \mathbf{P}_{5 / 2}$ & 2592.1 & $0.33 \pm 0.07$ & 0.12 & 0.045 & & & & \\
\hline \multirow{4}{*}{$5 s^{2} 5 f^{2} \mathbf{F}_{5 / 2}$} & $5 \mathrm{~s}^{2} 5 \mathrm{~d}^{2} \mathbf{D}_{3 / 2}$ & 3537.6 & $0.36 \pm 0.07$ & 1.7 & 2.3 & & & & \\
\hline & $5 \mathrm{~s}^{2} 5 \mathrm{~d}^{2} \mathbf{D}_{5 / 2}$ & 3620.1 & $0.62 \pm 0.10$ & 0.11 & 0.16 & & & & \\
\hline & $5 s 5 p^{2}{ }^{2} D_{3 / 2}$ & 2448.9 & $2.2 \pm 0.5$ & 6.8 & 4.7 & 3.7 & & & \\
\hline & $5 s 5 p^{22} D_{5 / 2}$ & 2486.7 & $1.0 \pm 0.2$ & 0.48 & 0.28 & & & & \\
\hline \multirow[t]{2}{*}{$5 s^{2} 5 f^{2} \mathbf{F}_{7 / 2}$} & $5 \mathrm{~s}^{2} 5 \mathrm{~d}^{2} \mathbf{D}_{5 / 2}$ & 3620.5 & $0.17 \pm 0.04$ & 1.7 & 2.4 & & & & $0.240 \%$ \\
\hline & $5 s 5 p^{2}{ }^{2} D_{5 / 2}$ & 2487.0 & $2.7 \pm 0.5$ & 7.3 & 4.2 & 5.5 & & & \\
\hline \multirow{2}{*}{$5 s 5 p^{2}{ }^{4} \mathbf{P}_{1 / 2}$} & $5 s^{2} 5 p^{2} \mathbf{P}_{1 / 2}$ & 2151.5 & $0.011 \pm 0.008$ & 0.12 & 0.06 & & & & \\
\hline & $5 \mathrm{~s}^{2} 5 \mathrm{p}^{2} \mathbf{P}_{3 / 2}$ & 2368.3 & $0.055 \pm 0.010$ & 0.034 & 0.008 & 0.044 & & & \\
\hline $5 \mathrm{~s} 5 \mathrm{p}^{2}{ }^{4} \mathbf{P}_{3 / 2}$ & $5 \mathrm{~s}^{2} 5 \mathrm{p}^{2} \mathbf{P}_{3 / 2}$ & 2266.0 & $0.0031 \pm 0.001$ & 0.033 & 0.030 & & & & \\
\hline $5 \mathrm{~s} 5 \mathrm{p}^{2}{ }^{4} \mathbf{P}_{5 / 2}$ & $5 s^{2} 5 p^{2} \mathbf{P}_{3 / 2}$ & 2150.9 & $0.0030 \pm 0.001$ & 0.076 & 0.047 & & & & \\
\hline
\end{tabular}

${ }^{\mathrm{a}}$ McCormick and Sawyer [1].

${ }^{\mathrm{b}}$ Wujec and Weniger [3].

${ }^{\mathrm{c}}$ Wujec and Musielok [2].

${ }^{\mathrm{d}}$ Miller et al. [4]. 


\subsection{Experimental transition probabilities.}

The transition probabilities of lines with origin in the $5 \mathrm{~s}^{2} 4 \mathrm{f}$ ${ }^{2} \mathrm{~F}_{5 / 2}$ of Sn II obtained from lifetime and branching-ratio measurements are shown in the third column of Table V.

The experimental errors for these values are the results of statistical uncertainties, errors in the lifetime measurements and uncertainties in the spectral response determination; data given by other authors have been included for comparison.

The transition probabilities obtained from plasma temperature for $36 \mathrm{Sn}$ II lines with wavelengths in the range between 2100-6900 $\AA$ are displayed in the third column of Table VI. Quoted errors have been calculated as the quadratic additions of the statistical uncertainties and the temperature errors. The first and second columns list the transitions and the corresponding wavelengths.

In column four we display the absolute transition probabilities obtained from HFR calculations including configuration interactions. In the right side of the column we displays the theoretical values resulting from least-square fitting. Data given by others authors have been included in the columns to the right in the table for comparison. These values are in suitable agreement with the results present in this work.

\section{Acknowledgements}

It is a pleasure to acknowledge stimulating discussions with Professor J. Campos. This work was supported by the Dpto. de Física Atómica, Molecular y Nuclear (Fac. de CC. Físicas. U.C.M., Spain). 